Article

\title{
Some Liouville Theorems on Finsler Manifolds
}

\author{
Minqiu Wang ${ }^{1}$ and Songting Yin ${ }^{2,3, *}$ \\ 1 Department of Mathematics and Physics, Hefei University, Hefei 230601, China; wmq@hfuu.edu.cn \\ 2 Department of Mathematics and Computer Science, Tongling University, Tongling 244000, China \\ 3 Key Laboratory of Applied Mathematics (Putian University), Fujian Province University, \\ Putian 351100, China \\ * Correspondence: yst419@163.com
}

Received: 22 January 2019; Accepted: 11 April 2019; Published: 15 April 2019

check for updates

\begin{abstract}
We give some Liouville type theorems of $L^{p}$ harmonic (resp. subharmonic, superharmonic) functions on a complete noncompact Finsler manifold. Using the geometric relationship between a Finsler metric and its reverse metric, we remove some restrictions on the reversibility. These improve the recent literature (Zhang and Xia, 2014).
\end{abstract}

Keywords: harmonic function; Finsler manifold; Liouville theorem; reversibility

MSC: Primary 53C60; Secondary 53B40

\section{Introduction}

As is well known, Liouville theorems play an important role in analyzing the underlying manifolds. The classical Liouville theorem states that any nonnegative (or bounded) harmonic function on $\mathbb{R}^{n}$ must be constant. Up to now, there are many generalizations studied on complete Riemannian manifolds. Yau [1,2] proved that any positive (or bounded) harmonic function on a complete Riemannian manifold with nonnegative Ricci curvature must be constant and there are no nonnegative $L^{p}$ subharmonic functions on such a Riemannian manifold for $p \in(1, \infty)$. Yau's results were then generalized by Sturm and Schoen, etc. See [3-5] and references therein. For general symmetric diffusion operators, $\mathrm{Li}$ [6] extended various Liouville theorems as above.

Recently, Zhang-Xia [7], Yin-He [8] and Yin-Zhang [9] extended the above Liouville theorems in the Finsler setting. Notice that, in $[7,8]$, the Finsler manifolds discussed must have finite reversibility. In this paper, we show that this restriction can be removed. Specifically, we obtain the following results.

Theorem 1. Let $(M, F, d \mu)$ be an n-dimensional forward complete noncompact Finsler manifold. If a positive function $u \in W_{l o c}^{2,2}(M) \cap C^{1, \alpha}(M) \cap C^{\infty}\left(M_{u}\right)$ on $M$ satisfies $\Delta \log u \geq 0$ on $M_{u}$ and

$$
\limsup _{r \rightarrow \infty} \frac{r^{2}}{V_{1}(r)}=\infty
$$

where $M_{u}=\{x \in M \mid d u(x) \neq 0\}$, then $u$ is a constant. In particular, if $u \in L^{1}(M)$ and $\Delta \log u \geq 0$ on $M_{u}$, then $u$ is a constant.

Theorem 2. Let $(M, F, d \mu)$ be an n-dimensional complete noncompact Finsler manifold. Assume that

$$
\int_{1}^{\infty} \frac{r}{V_{p}(r)} d r=\infty
$$


1. If $p \in(-\infty, 1)$ and $u \in W_{l o c}^{2,2}(M) \cap C^{1, \alpha}(M) \cap C^{\infty}\left(M_{u}\right)$ is a nonnegative superharmonic function on $M$, then $u$ is a constant.

2. If $p \in(1, \infty)$ and $u \in W_{\text {loc }}^{2,2}(M) \cap C^{1, \alpha}(M) \cap C^{\infty}\left(M_{u}\right)$ is a nonnegative subharmonic function on $M$, then $u$ is a constant.

Here, $V_{p}(r), p \in \mathbb{R}$ is defined in (2) below, and some important concepts such as Finsler metric, Finsler Laplacian and harmonic (resp. subharmonic, superharmonic) functions will be given in Section 2, respectively.

Remark 1. If the Finsler manifold is compact, then, by the divergence theorem, we can prove all harmonic (resp. subharmonic, superharmonic) functions are constant. Theorem 1 can be regarded as a generalization of Theorem 1 in [2] when $p=1$. If $(M, F, d \mu)$ is a Riemannian metric measure space, then Theorem 2 is exactly Theorem 1 in [5] or Theorem 13.1 in [10].

Remark 2. In comparison with [7], the condition on the reversibility is deleted in theorems above. There are many Finsler manifolds with infinity reversibility. Consider the Randers metric in $\mathbb{B}^{3}(1)$

$$
F(x, y)=\sqrt{\left(y^{1}\right)^{2}+\left(y^{2}\right)^{2}+\left(y^{3}\right)^{2}}+|x| y^{1}, \quad|x|<1,
$$

where $|\cdot|$ denotes the standard Euclid norm. Then the reversibility

$$
\lambda_{F}(x)=\frac{1+|x|}{1-|x|} \rightarrow+\infty, \quad \text { if } x \rightarrow \partial \mathbb{B}^{3}(1) .
$$

The geometric quantities between $F$ and its reverse metric $\overleftarrow{F}$ have many important relationships. For example,

1. $F(-d f)=\overleftarrow{F}(d f), \overleftarrow{\nabla} f=-\nabla(-f), \overleftarrow{\Delta} f=-\Delta(-f)$

2. A forward (backward) distance function w.r.t. $F$ is a backward (forward) distance function w.r.t. $\overleftarrow{F}$, and vice versa.

3. A forward (backward) geodesic ball w.r.t. $F$ is a backward (forward) geodesic ball w.r.t. $\overleftarrow{F}$, and vice versa.

4. If $f$ is a superharmonic (subharmonic) function w.r.t. $\Delta$, then $-f$ is a subharmonic (superharmonic) function w.r.t. $\overleftarrow{\Delta}$, and vice versa.

To give a more elaborate improvement, we use these relations and thus avoid employing the reversibility. The remainder of the approaches adopted are similar to Zhang-Xia's paper [7]. See also in [2,5,10] for the Riemannian case.

The contents of the paper are arranged as follows. In Section 2, some fundamental concepts which are necessary for the present paper are given, and some lemmas are contained. In Section 3, we prove the main theorems and give some corollaries.

\section{Preliminaries}

Let $M$ be an $n$-dimensional smooth manifold and $\pi: T M \rightarrow M$ be the natural projection from the tangent bundle $T M$. Let $(x, y)$ be a point of $T M$ with $x \in M, y \in T_{x} M$, and let $\left(x^{i}, y^{i}\right)$ be the local coordinates on $T M$ with $y=y^{i} \partial / \partial x^{i}$. A Finsler metric on $M$ is a function $F: T M \rightarrow[0,+\infty)$ satisfying the following properties:

(i) Regularity: $F(x, y)$ is smooth in $T M \backslash 0$;

(ii) Positive homogeneity: $F(x, \lambda y)=\lambda F(x, y)$ for $\lambda>0$; 
(iii) Strong convexity: The fundamental quadratic form

$$
g:=g_{i j}(x, y) d x^{i} \otimes d x^{j}, \quad g_{i j}:=\frac{1}{2}\left[F^{2}\right]_{y^{i} y^{j}}
$$

is positive definite.

Let $X=X^{i} \frac{\partial}{\partial x^{i}}$ be a vector field. Then, the covariant derivative of $X$ by $v \in T_{x} M$ with reference vector $w \in T_{x} M \backslash 0$ is defined by

$$
D_{v}^{w} X(x):=\left\{v^{j} \frac{\partial X^{i}}{\partial x^{j}}(x)+\Gamma_{j k}^{i}(w) v^{j} X^{k}(x)\right\} \frac{\partial}{\partial x^{i}},
$$

where $\Gamma_{j k}^{i}$ denote the coefficients of the Chern connection.

For a smooth function $u$, the gradient vector of $u$ is

$$
\nabla u:=\ell^{-1}(d u),
$$

where $\ell: T M \rightarrow T^{*} M$ is Legendre transformation defined as

$$
\ell(y):= \begin{cases}g_{y}(y, \cdot), & y \in T M \backslash 0 \\ 0, & y=0\end{cases}
$$

Let $V=V^{i} \frac{\partial}{\partial x^{i}}$ be a smooth vector field on $M$. The divergence of $V$ with respect to an arbitrary smooth volume form $d \mu$ is defined by

$$
\operatorname{div} V:=\sum_{i=1}^{n}\left(\frac{\partial V^{i}}{\partial x^{i}}+V^{i} \frac{\partial \Phi}{\partial x^{i}}\right)
$$

where $d \mu=e^{\Phi} d x$. Then, the Finsler Laplacian of $u$ can be defined by

$$
\Delta u:=\operatorname{div}(\nabla u) .
$$

Since $\Delta u$ is undefined at $x$ where $d u(x)=0$, the definition can be viewed in distributional sense. That is, for $u \in W^{1,2}(M)$,

$$
\int_{M} \varphi \Delta u d \mu=-\int_{M} d \varphi(\nabla u) d \mu, \quad \forall \varphi \in C_{0}^{\infty}(M) .
$$

We note here that since the gradient operator $\nabla$ is not linear operator in general, the Finsler Laplacian is quite a bit different from the Riemannian Laplacian. Given a vector field $V$ such that $V \neq 0$ on $M_{u}$, where $M_{u}:=\{x \in M \mid d u(x) \neq 0\}$, the weighted gradient vector and the weighted Laplacian on the weighted Riemannian manifold $\left(M, g_{V}\right)$ are defined by

$$
\nabla^{V} u:=\left\{\begin{array}{ll}
g^{i j}(V) \frac{\partial u}{\partial x^{j}} \frac{\partial}{\partial x^{i}}, & \text { on } \quad M_{u}, \\
0, & \text { on } \quad M \backslash M_{u},
\end{array} \quad \Delta^{V} u=\operatorname{div}\left(\nabla^{V} u\right) .\right.
$$

It follows that $\nabla^{\nabla u} u=\nabla u, \quad \Delta^{\nabla u} u=\Delta u$.

Let $u$ be a positive harmonic function on $M, \Delta u=0$. It was proved that $u \in W_{\text {loc }}^{2,2}(M) \cap$ $C^{1, \alpha}(M) \cap C^{\infty}\left(M_{u}\right)$ (see [11]). We say that $u \in W_{l o c}^{2,2}(M) \cap C^{1, \alpha}(M) \cap C^{\infty}\left(M_{u}\right)$ is a subharmonic (resp. superharmonic) function on $M$ if $\Delta u \geq 0$ (resp. $\Delta u \leq 0$ ). In a weak sense, $u$ is a subharmonic (resp. superharmonic) function in $M$ if, for any positive function $\varphi \in C_{0}^{\infty}(M)$, it holds

$$
\int_{M} \varphi \Delta u d \mu \geq(\text { resp. } \leq) 0
$$


Let $(M, F)$ be a Finsler $n$-manifold. Fix a point $x_{0} \in M$. We denote a forward (resp. backward) geodesic ball of radius $r$ with center at $x_{0}$ by $B_{x_{0}}^{+}(r)\left(\operatorname{resp} . B_{x_{0}}^{-}(r)\right)$.

Lemma 1. Let $(M, F)$ be a Finsler n-manifold and $x_{0} \in M$. Then, there exists a function defined by

$$
\varphi(x)= \begin{cases}1, & x \in B_{x_{0}}^{+}(R) ; \\ 0, & x \in M \backslash B_{x_{0}}^{+}(2 R)\end{cases}
$$

such that

$$
F^{*}(-d \varphi) \leq \frac{C}{R}
$$

where $C$ is a positive constant.

Proof. Let $\omega(t)$ be a smooth function on the real line with $0 \leq \omega(t) \leq 1$ and $\omega^{\prime}(t) \leq 0$ such that

$$
\omega(t)= \begin{cases}1, & 0 \leq t \leq 1, \\ 0, & t \geq 2\end{cases}
$$

Clearly, $\left|\omega^{\prime}(t)\right| \leq C$, where $C$ is some positive constant. Define

$$
\varphi(x)=\omega\left(\frac{r(x)}{R}\right)
$$

where $r(x)=d_{F}\left(x_{0}, x\right)$ is the distance function form $x_{0}$. Then,

$$
\begin{aligned}
F^{*}(-d \varphi) & =F^{*}\left(-\frac{\omega^{\prime}}{R} d r\right)=\frac{\left|\omega^{\prime}\right|}{R} F^{*}(d r) \\
& =\frac{\left|\omega^{\prime}\right|}{R} \leq \frac{C}{R} \quad \text { a.e. on } \quad M .
\end{aligned}
$$

Notice that $\varphi \in C_{0}^{\infty}\left(B_{x_{0}}^{+}(2 R) \backslash\left(\left\{x_{0}\right\} \cup \operatorname{cut}\left(x_{0}\right)\right)\right)$ and it is differentiable almost everywhere on $B_{x_{0}}^{+}(2 R)$ with bounded differential. Since a subharmonic (resp. superharmonic) function $u$ belongs to $W_{\text {loc }}^{2,2}(M) \cap C^{1, \alpha}(M) \cap C^{\infty}\left(M_{u}\right)$, and $\Delta u=0$ a.e. on $M \backslash M_{u}$ (Lemma 3.5 in [12]), we find the Formula (1) still holds for this $\varphi$.

\section{Proof of the Main Theorems}

For any nonnegative function $u$, set

$$
V_{p}(r)= \begin{cases}\int_{B_{x_{0}}^{+}(r)} u^{p} d \mu, & p \geq 1 \\ \int_{B_{x_{0}}^{-}(r)} u^{p} d \mu, & p<1 .\end{cases}
$$

Note that $V_{p}(r)=\operatorname{vol}^{d \mu}\left(B_{x_{0}}^{-}(r)\right):=V(r)$ if $p=0$.

Proof of Theorem 1. Set $v=\sqrt{u}$. Then, in $M_{u}=M_{v}$, one obtains

$$
0 \leq \frac{1}{2} \Delta \log u=\Delta \log v=\operatorname{div}\left(\frac{\nabla v}{v}\right)=\frac{\Delta v}{v}-\frac{F(\nabla v)^{2}}{v^{2}}
$$

which gives

$$
v \Delta v \geq F(\nabla v)^{2} \geq 0
$$


Let $\varphi$ be the function defined in Lemma 1. Then, it is differentiable almost everywhere on $\left(B_{x_{0}}^{+}(2 R)\right)$ with a bounded differential. Note that $\Delta v=0$ a.e. on $M \backslash M_{v}$ from Lemma 3.5 in [12]. Thus, by the divergence theorem, we have

$$
\begin{aligned}
\int_{B_{x_{0}}^{+}(2 R) \cap M_{v}} \varphi^{2} v \Delta v d \mu & =\int_{B_{x_{0}}^{+}(2 R)} \varphi^{2} v \Delta v d \mu=-\int_{B_{x_{0}}^{+}(2 R)} d\left(\varphi^{2} v\right)(\nabla v) d \mu \\
& =-\int_{B_{x_{0}}^{+}(2 R)} \varphi^{2} F(\nabla v)^{2} d \mu-2 \int_{B_{x_{0}}^{+}(2 R)} \varphi v d \varphi(\nabla v) d \mu \\
& \leq-\int_{B_{x_{0}}^{+}(2 R)} \varphi^{2} F(\nabla v)^{2} d \mu+2 \int_{B_{x_{0}}^{+}(2 R)} \varphi v F^{*}(-d \varphi) F(\nabla v) d \mu .
\end{aligned}
$$

Therefore,

$$
\begin{aligned}
\int_{B_{x_{0}}^{+}(2 R)} \varphi^{2} F(\nabla v)^{2} d \mu & \leq 2 \int_{B_{x_{0}}^{+}(2 R)} \varphi v F^{*}(-d \varphi) F(\nabla v) d \mu \\
& \leq 2\left(\int_{B_{x_{0}}^{+}(2 R)} \varphi^{2} F(\nabla v)^{2} d \mu\right)^{\frac{1}{2}}\left(\int_{B_{x_{0}}^{+}(2 R)} v^{2} F^{*}(-d \varphi)^{2} d \mu\right)^{\frac{1}{2}},
\end{aligned}
$$

which implies that

$$
\int_{B_{x_{0}}^{+}(2 R)} \varphi^{2} F(\nabla v)^{2} d \mu \leq 4 \int_{B_{x_{0}}^{+}(2 R)} v^{2} F^{*}(-d \varphi)^{2} d \mu
$$

By using Lemma 1 and the definition of $\varphi$, we deduce

$$
\int_{B_{x_{0}}^{+}(R)} \varphi^{2} F(\nabla v)^{2} d \mu \leq \frac{4 C^{2}}{R^{2}} \int_{B_{x_{0}}^{+}(2 R)} v^{2} d \mu=\frac{16 C^{2}}{(2 R)^{2}} \int_{B_{x_{0}}^{+}(2 R)} u d \mu \quad=16 C^{2} \frac{V_{1}(2 R)}{(2 R)^{2}} .
$$

Letting $R \rightarrow \infty$, it follows from $\limsup _{r \rightarrow \infty} \frac{r^{2}}{V_{1}(r)}=\infty$ that $F(\nabla v)=0$ everywhere. Since $M$ is connected, $v$ is a constant on $M$ and so is $u$.

Proof of Theorem 2. Without loss of generality, we might as well assume $u>0$. Otherwise, we can replace it by $\tilde{u}=u+\varepsilon>0$ for some positive number $\varepsilon$. We first prove (2) in Theorem 2. Let $x_{0}$ be a fixed point in $M$ and $r_{0}$ be a number with $0<r_{0}<R$. Define

$$
\psi(x)= \begin{cases}1, & x \in \bar{B}_{x_{0}}^{+}\left(r_{0}\right) ; \\ 0, & x \in M \backslash B_{x_{0}}^{+}(R),\end{cases}
$$

with $\psi(x) \in C_{0}^{\infty}\left(B_{x_{0}}^{+}(R) \backslash\left(\left\{x_{0}\right\} \cup \operatorname{cut}\left(x_{0}\right)\right)\right)$ satisfying

$$
F^{*}(-d \psi) \leq \frac{C}{R} \leq \frac{C}{r_{0}}:=\widehat{C} .
$$


Using the divergence theorem and similar arguments above, we have

$$
\begin{aligned}
& (p-1) \int_{B_{x_{0}}^{+}(R) \cap M_{u}} u^{p-1} \psi^{2} \Delta u d \mu \\
= & (p-1) \int_{B_{x_{0}}^{+}(R)} u^{p-1} \psi^{2} \Delta u d \mu \\
= & -(p-1) \int_{B_{x_{0}}^{+}(R)} d\left(u^{p-1} \psi^{2}\right)(\nabla u) d \mu \\
= & -(p-1)^{2} \int_{B_{x_{0}}^{+}(R)} u^{p-2} \psi^{2} F(\nabla u)^{2} d \mu-2(p-1) \int_{B_{x_{0}}^{+}(R)} u^{p-1} \psi d \psi(\nabla u) d \mu .
\end{aligned}
$$

Set $v=u^{\frac{p}{2}}$. Then, (4) becomes

$$
\begin{aligned}
& (p-1) \int_{B_{x_{0}}^{+}(R)} u^{p-1} \psi^{2} \Delta u d \mu \\
= & -4\left(1-\frac{1}{p}\right)^{2} \int_{B_{x_{0}}^{+}(R)} \psi^{2} F(\nabla v)^{2} d \mu-4\left(1-\frac{1}{p}\right) \int_{B_{x_{0}}^{+}(R)} v \psi d \psi(\nabla v) d \mu .
\end{aligned}
$$

From the conditions in Theorem 2 and (3), it follows that

$$
\begin{aligned}
& \left(1-\frac{1}{p}\right)^{2} \int_{B_{x_{0}}^{+}(R)} \psi^{2} F(\nabla v)^{2} d \mu \\
\leq & -\left(1-\frac{1}{p}\right) \int_{B_{x_{0}}^{+}(R) \backslash \bar{B}_{x_{0}}^{+}\left(r_{0}\right)} v \psi d \psi(\nabla v) d \mu \\
\leq & \left(\int_{B_{x_{0}}^{+}(R) \backslash \bar{B}_{x_{0}}^{+}\left(r_{0}\right)} v^{2} F^{*}(-d \psi)^{2} d \mu\right)^{\frac{1}{2}}\left(\left(1-\frac{1}{p}\right)^{2} \int_{B_{x_{0}}^{+}(R) \backslash \bar{B}_{x_{0}}^{+}\left(r_{0}\right)} \psi^{2} F(\nabla v)^{2} d \mu\right)^{\frac{1}{2}} \\
\leq & \widehat{C}\left(\int_{B_{x_{0}}^{+}(R) \backslash \bar{B}_{x_{0}}^{+}\left(r_{0}\right)} u^{p} d \mu\right)^{\frac{1}{2}}\left(\left(1-\frac{1}{p}\right)^{2} \int_{B_{x_{0}}^{+}(R) \backslash \bar{B}_{x_{0}}^{+}\left(r_{0}\right)} \psi^{2} F(\nabla v)^{2} d \mu\right)^{\frac{1}{2}} \\
= & \widehat{C}\left(V_{p}(R)-V_{p}\left(r_{0}\right)\right)^{\frac{1}{2}}\left(\left(1-\frac{1}{p}\right)^{2} \int_{B_{x_{0}}^{+}(R) \backslash \bar{B}_{x_{0}}^{+}\left(r_{0}\right)} \psi^{2} F(\nabla v)^{2} d \mu\right)^{\frac{1}{2}} .
\end{aligned}
$$

Let

$$
G(r)=\left(1-\frac{1}{p}\right)^{2} \int_{B_{x_{0}}^{+}(r)} F(\nabla v)^{2} d \mu .
$$

Then, by similar arguments as in [7], we can also reach

$$
\frac{1}{G\left(r_{0}\right)}-\frac{1}{G(R)} \geq \frac{1}{\widehat{C}^{2}} \frac{\left(R-r_{0}\right)^{2}}{V_{p}(R)-V_{p}\left(r_{0}\right)} .
$$

For fixed $r_{0}$, taking $R_{k}=2^{k} r_{0}, k \in N^{+}$, we have

$$
\begin{aligned}
\frac{1}{G\left(r_{0}\right)} & \geq \frac{1}{G\left(R_{n}\right)}+\frac{1}{\widehat{C}^{2}} \sum_{k=1}^{n} \frac{\left(R_{k}-R_{k-1}\right)^{2}}{V_{p}\left(R_{k}\right)-V_{p}\left(R_{k-1}\right)} \geq \frac{1}{4 \widehat{C}^{2}} \sum_{k=1}^{n} \frac{\left(R_{k}\right)^{2}}{V_{p}\left(R_{k}\right)} \\
& =\frac{1}{8 \widehat{C}^{2}} \sum_{k=1}^{n} \frac{2 R_{k}^{2}}{V_{p}\left(R_{k}\right)}=\frac{1}{8 \widehat{C}^{2}} \sum_{k=1}^{n} \frac{2^{k+1} r_{0}}{V_{p}\left(2^{k} r_{0}\right)} \times 2^{k} r_{0} \\
& \geq \frac{1}{8 \widehat{C}^{2}} \sum_{k=1}^{n} \int_{2^{k} r_{0}}^{2^{k+1} r_{0}} \frac{r}{V_{p}(r)} d r .
\end{aligned}
$$


Letting $n \rightarrow \infty$, we have

$$
\frac{1}{G\left(r_{0}\right)} \geq \frac{1}{8 \widehat{C}^{2}} \int_{2 r_{0}}^{\infty} \frac{r}{V_{p}(r)} d r=\infty,
$$

which means that

$$
\int_{B_{x_{0}}^{+}\left(r_{0}\right)} F(\nabla v)^{2} d \mu=0 .
$$

Therefore, by arbitrariness of $r_{0}$, we conclude that $v$ must be constant on $M$ and so is $u$.

Now, we are to prove (1) according to the cases $0<p<1, p<0$ and $p=0$, respectively.

Case I: $0<p<1$.

Let $x_{0}$ and $r_{0}$ be as above. Define

$$
\psi(x)= \begin{cases}1, & x \in \bar{B}_{x_{0}}^{-}\left(r_{0}\right) ; \\ 0, & x \in M \backslash B_{x_{0}}^{-}(R),\end{cases}
$$

with $\psi(x) \in C_{0}^{\infty}\left(B_{x_{0}}^{-}(R) \backslash\left(\left\{x_{0}\right\} \cup \operatorname{cut}\left(x_{0}\right)\right)\right)$ satisfying

$$
F^{*}(d \psi) \leq \frac{C}{R} \leq \frac{C}{r_{0}}:=\widehat{C} .
$$

By similar arguments, we also obtain (5) for the backward geodesic ball. The remainder of the proof is the same as above.

Case II: $p<0$.

Set $v=-u^{\frac{p}{2}}$. Then,

$$
F(\nabla v)=-\frac{p}{2} u^{\frac{p}{2}-1} F(\nabla u) .
$$

Let $\psi$ be a function as in Case I. We can also obtain

$$
\begin{aligned}
0 & \leq(p-1) \int_{B_{x_{0}}^{-}(R)} u^{p-1} \psi^{2} \Delta u d \mu \\
& =-(p-1)^{2} \int_{B_{x_{0}}^{-}(R)} u^{p-2} \psi^{2} F(\nabla u)^{2} d \mu-2(p-1) \int_{B_{\bar{x}_{0}}^{-}(R)} u^{p-1} \psi d \psi(\nabla u) d \mu . \\
& =-4\left(1-\frac{1}{p}\right)^{2} \int_{B_{x_{0}}^{-}(R)} \psi^{2} F(\nabla v)^{2} d \mu-4\left(1-\frac{1}{p}\right) \int_{B_{x_{0}}^{-}(R)} v \psi d \psi(\nabla v) d \mu .
\end{aligned}
$$

Therefore, we have

$$
\begin{aligned}
& \left(1-\frac{1}{p}\right)^{2} \int_{B_{x_{0}}^{-}(R)} \psi^{2} F(\nabla v)^{2} d \mu \\
\leq & -\left(1-\frac{1}{p}\right) \int_{B_{x_{0}}^{-}(R) \backslash \bar{B}_{x_{0}}^{-}\left(r_{0}\right)} v \psi d \psi(\nabla v) d \mu \\
\leq & \left(\int_{B_{x_{0}}^{-}(R) \backslash \bar{B}_{\bar{x}_{0}}^{-}\left(r_{0}\right)} v^{2} F^{*}(d \psi)^{2} d \mu\right)^{\frac{1}{2}}\left(\left(1-\frac{1}{p}\right)^{2} \int_{B_{x_{0}}^{-}(R) \backslash \bar{B}_{x_{0}}^{-}\left(r_{0}\right)} \psi^{2} F(\nabla v)^{2} d \mu\right)^{\frac{1}{2}} \\
= & \widehat{C}\left(V_{p}(R)-V_{p}\left(r_{0}\right)\right)^{\frac{1}{2}}\left(\left(1-\frac{1}{p}\right)^{2} \int_{B_{x_{0}}^{-}(R) \backslash \bar{B}_{x_{0}}^{-}\left(r_{0}\right)} \psi^{2} F(\nabla v)^{2} d \mu\right)^{\frac{1}{2}} .
\end{aligned}
$$

Then, by the same argument as above, one obtains that $u$ is constant. 
Case III: $p=0$.

For every $k \in \mathbb{R}^{+}$, set

$$
u_{k}= \begin{cases}k, & u \geq k ; \\ u, & u<k .\end{cases}
$$

In what follows, we will follow the arguments in [10] (p. 178) with some modifications. Let $\beta$ be a symmetric, convex, and bounded smooth function with $\left|\beta^{\prime}\right|<1$ and $|s|<\beta<\epsilon+|s|$, where $0<\epsilon<1$ is such that $u-\epsilon>0$. Define

$$
\tilde{u}_{k}=\frac{u+k}{2}-\frac{\beta(u-k)}{2} .
$$

Then, for any positive integer $k$, it holds that $\tilde{u}_{k}>\frac{u+k}{2}-\frac{\epsilon+|u-k|}{2}>0$. Moreover, $\tilde{u}_{k}$ is a superharmonic function in a weak sense. Indeed, by definition, we have $d \tilde{u}_{k}=\frac{1}{2}\left(1-\beta^{\prime}\right) d u$, which yields $\nabla \tilde{u}_{k}=$ $\frac{1}{2}\left(1-\beta^{\prime}\right) \nabla u$ by Legendre transformation. As $\tilde{u}_{k} \in H_{l o c}^{2}$ and thus $\Delta \tilde{u}_{k}=0$ a.e. on $M \backslash M_{u}$, for $\psi$ defined in Case I, we have

$$
\begin{aligned}
2 \int_{B_{x_{0}}^{-}(R)} \psi \Delta \tilde{u}_{k} d \mu & =-2 \int_{B_{x_{0}}^{-}(R)} d \psi\left(\nabla \tilde{u}_{k}\right) d \mu=-\int_{B_{x_{0}}^{-}(R)}\left(1-\beta^{\prime}\right) d \psi(\nabla u) d \mu \\
& =-\int_{B_{x_{0}}^{-}(R)} d\left[\left(1-\beta^{\prime}\right) \psi\right](\nabla u) d \mu-\int_{B_{x_{0}}^{-}(R)} \beta^{\prime \prime} \psi F(\nabla u)^{2} d \mu \\
& \leq-\int_{B_{x_{0}}^{-}(R)} d\left[\left(1-\beta^{\prime}\right) \psi\right](\nabla u) d \mu=\int_{B_{x_{0}}^{-}(R)}\left(1-\beta^{\prime}\right) \psi \Delta u d \mu \\
& \leq 0 .
\end{aligned}
$$

The last step holds because $\left(1-\beta^{\prime}\right) \psi$ is differentiable almost everywhere on $B_{x_{0}}^{-}(R)$ with bounded differential, and $u$ is superharmonic. Moreover, $\tilde{u}_{k}$ is smooth on the open subset $M_{u}$ and is also superharmonic, in the classical sense, on $M_{u}$. Notice that $\psi$ is differentiable almost everywhere on $B_{x_{0}}^{-}(R)$ with bounded differential. Hence, by similar arguments, we can also obtain (4) for $\tilde{u}_{k}$ on $B_{x_{0}}^{-}(R)$ as in case I. Set $v_{k}=\tilde{u}_{k}^{\frac{q}{2}}$ for any $q \in(0,1)$. Then we have (5) as follows:

$$
\begin{aligned}
& \left(1-\frac{1}{q}\right)^{2} \int_{B_{x_{0}}^{-}(R)} \psi^{2} F\left(\nabla v_{k}\right)^{2} d \mu \\
\leq & \widehat{C}\left(\int_{B_{x_{0}}^{-}(R) \backslash \bar{B}_{x_{0}}^{-}\left(r_{0}\right)} v_{k}^{2}\right)^{\frac{1}{2}}\left(\left(1-\frac{1}{q}\right)^{2} \int_{B_{\bar{x}_{0}}^{-}(R) \backslash \bar{B}_{x_{0}}^{-}\left(r_{0}\right)} \psi^{2} F\left(\nabla v_{k}\right)^{2} d \mu\right)^{\frac{1}{2}} \\
= & \widehat{C}\left(V_{q}(R)-V_{q}\left(r_{0}\right)\right)^{\frac{1}{2}}\left(\left(1-\frac{1}{q}\right)^{2} \int_{B_{\bar{x}_{0}}^{-}(R) \backslash \bar{B}_{\bar{x}_{0}}^{-}\left(r_{0}\right)} \psi^{2} F\left(\nabla v_{k}\right)^{2} d \mu\right)^{\frac{1}{2}} .
\end{aligned}
$$

On the other hand, note that $\tilde{u}_{k} \leq k$, and thus

$$
\int_{B_{x_{0}}^{-}(R)} \tilde{u}_{k}^{q} d \mu \leq \int_{B_{x_{0}}^{-}(R)} k^{q} d \mu=k^{q} V(R),
$$

which implies that

$$
\int_{1}^{\infty} \frac{r}{V_{q}(r)} d r=\infty
$$

Then by the same discussion in the proof of (2) and Case I of (1), we show that this $\tilde{u}_{k}$ is constant. Take then a sequence $\beta_{n}$ (such that each $\beta_{n}$ satisfies the same properties as $\beta$ ) uniformly converging to the absolute value function. Every $\tilde{u}_{k, n}$ is then constant. These constants are bounded (they are in $(0, k)$ ). Thus, up to pass to a subsequence $\tilde{u}_{k, n}$ converges uniformly to $u_{k}$ and to a constant at the same time. Hence, $u_{k}$ must be constant. $k$ being arbitrary, $u$ is also constant. 
Using Theorem 2, we can reach the following corollaries which extend Theorem 3 in [2] and Corollary 1 in [13], respectively.

Corollary 1. Let $(M, F, d \mu)$ and $u$ be as in Theorem 2.

1. If $p \in(-\infty, 1)$, then every nonnegative superharmonic function $u \in L^{p}(M)$ is a constant. In particular, if $\mathrm{vol}^{d \mu}(M)<\infty$, then every nonnegative superharmonic function on $M$ is a constant.

2. If $p \in(1, \infty)$, then every nonnegative subharmonic function $u \in L^{p}(M)$ is a constant.

Corollary 2. Let $(M, F, d \mu)$ and $u$ be as in Theorem 2 and $u$ be a nonnegative superharmonic function. If, for a sequence $r_{k} \rightarrow+\infty$,

$$
V\left(r_{k}\right) \leq C r_{k}^{2}
$$

then $u$ is a constant, where $C$ is a positive constant.

Author Contributions: Y.S. suggeated to study this topic. W.M. checked the calculations and polished the draft. Funding: This project is supported by EYTVSP (No.gxfx2017095), FMDEP (No.2018xs03), AHNSF (No.160808 5MA03), KLAMFJPU (No. SX201805) and TLXYXM (No. 2018tlxyzd02).

Acknowledgments: The authors like to sincerely thank the Academic Editor for very valuable and helpful comments and suggestions.

Conflicts of Interest: The authors declare no conflict of interest.

\section{References}

1. Yau, S.T. Harmonic functions on complete Riemannian manifolds. Comm. Pure Appl. Math. 1975, 28, 201-208.

2. Yau, S.T. Some function theoretic properties of complete Riemannian manifolds and their applications to geometry. Indiana Univ. Math. J. 1976, 25, 659-670. [CrossRef]

3. $\mathrm{Li}$, P. Uniqueness of $\mathrm{L} 1$ solutions for the Laplace equation and the heat equation on Riemannian manifolds. J. Diff. Geom. 1985, 20, 447-457. [CrossRef]

4. Li, P.; Schoen, R. $L^{p}$ and mean value properties of subharmonic functions on Riemannian manifolds. Acta Math. 1984, 153, 279-301. [CrossRef]

5. Sturm, K.T. Analysis on local Dirichlet spaces, I. recurrence, conservativeness and $L^{p}$-liouville properties. J. Reine Angew. Math. 1994, 456, 173-196.

6. Li, X. Liouville theorems for symmetric diffusion operators on complete Riemannian manifolds. J. Math. Pures Appl. 2005, 84, 1295-1361. [CrossRef]

7. Zhang, F.; Xia, Q. Some Liouville-type theorems for harmonic functions on Finsler manifolds. J. Math. Anal. Appl. 2014, 417, 979-995. [CrossRef]

8. Yin, S.; He, Q. A generalized Omori-Yau maximum principle in Finsler geometry. Nonlinear Anal. Theory Methods Appl. 2015, 128, 227-247. [CrossRef]

9. Yin, S.; Zhang, P. Remarks on Liouville-type theorems on complete noncompact Finsler manifolds. Revista de la Unión Matemática Argentina 2018, 59, 255-264. [CrossRef]

10. Grigor'yan, A. Analytic and geometric background of recurrence and nonexplosion of the brownian motion on Riemannian manifolds. Bull. Am. Math. Soc. (N.S.) 1999, 36, 135-249. [CrossRef]

11. Ohta, S.; Sturm, K.-T. Heat flow on Finsler manifolds. Commun. Pure Appl. Math. 2009, 62, 1386-1433. [CrossRef]

12. Ohta, S.; Sturm, K.T. Bochner-Weitzenbóck formula and Li-Yau estimates on Finsler manifolds. Adv. Math. 2014, 252, 429-448. [CrossRef]

13. Cheng, S.Y.; Yau, S.T. Differential equations on Riemannian manifolds and their geometric applications. Commu. Pure Appl. Math. 1975, 28, 333-354. [CrossRef]

(c) 2019 by the authors. Licensee MDPI, Basel, Switzerland. This article is an open access article distributed under the terms and conditions of the Creative Commons Attribution (CC BY) license (http:// creativecommons.org/licenses/by/4.0/). 